\title{
Meteorological Aspects of the Margaret River Fires of November 2011
}

\author{
$\underline{\text { Jeffrey D. Kepert }}^{\mathrm{a}, \mathrm{b}}$ and Robert J. B. Fawcett ${ }^{\mathrm{a}, \mathrm{b}}$ \\ a. Centre for Australian Weather and Climate Research, Melbourne, Australia \\ b. Bushfire Cooperative Research Centre, Melbourne, Australia \\ Email: $\underline{\text { J.Kepert@bom.gov.au }}$
}

\begin{abstract}
Early in the morning of Wednesday 23 November 2011, a fuel-reduction burn near Margaret River in southwest Western Australia increased dramatically in activity. The fire escaped control lines and burnt southwards along the coastal fringe, subsequently destroying 32 homes, including the historic Wallcliffe House, and damaging others. Satellite imagery showed a smoke plume of vastly greater extent than other fires in the area.

Here, we present a preliminary analysis of the meteorology of the event, based on very high resolution (400 m) simulations with the Bureau of Meteorology's ACCESS numerical weather prediction (NWP) system and available observations. We find that several mesoscale features likely contributed to the fire becoming or remaining active overnight. The first of these was a region of marked near-surface drying over and around the fire ground, contrary to the normal diurnal cycle and due to the advection of dry air from inland regions to the northeast of the fire. The second was the development of strong mountain-wave activity and downslope winds over the fire during the Tuesday night and extending into Wednesday morning, caused by a combination of a strengthening pressure gradient as a high pressure system passed to the south of the region, nocturnal cooling, and the local topography. The third was the development of a pronounced low-level jet, which provided a source of strong momentum for the mountain waves to bring to the surface.

During the day, while the fire was heading rapidly southwards under the influence of strong hot northerlies, the observed increase in activity around midday may have been due to a lengthy, coherent wake shed from hills to the north of the fire.

The essential dynamics of mountain waves are quite well known, although their occurrence on topography as low as here presents some forecasting difficulties, since the common assumption of inviscid flow cannot be made when the "mountain" is contained entirely within the boundary layer. Nevertheless, strong nocturnal downslope winds form a recognised part of the climate of both Perth and Adelaide, and likely occur in other regions also. Their influence on fire behaviour in Australia does not seem to have been widely documented before, although Sharples (2009) identifies one case. We expect that similar winds will occur again and recommend that managers of lee-slope fires exercise appropriate caution in the future, especially overnight and in the morning.
\end{abstract}

Keywords: Fire weather, downslope winds, low-level jet, ACCESS, high-resolution NWP 


\section{INTRODUCTION}

The weather, along with topography and fuel state, strongly influences bushfire behaviour. The influence of weather extends across multiple scales, ranging from the impact of long-term drought on fuel moisture, to the effects of small-scale flows induced by topography or land-sea contrasts. When all factors conspire towards elevated fire danger, ignitions may rapidly become uncontrollable and disaster may ensue, as occurred in Victoria on Black Saturday and similar occasions in the past. Paradoxically, such situations may not be the most difficult to identify as dangerous, precisely because all the factors agree. The most difficult may be those periods of apparently low to moderate fire danger, on a minority of which extreme, and unexpected, fire behaviour occurs.

The $23^{\text {rd }}$ of November 2011, near the town of Margaret River in south-western Western Australia, was one such event. Early in the morning, when fire activity would normally be expected to be at a minimum, a prescribed burn to the north of Margaret River escaped control lines and burnt rapidly southwards along the coast. Thirtytwo houses were destroyed (Keelty 2012), including the historic Wallcliffe House, and others damaged, while residents of coastal communities evacuated inland or to the beach. The rich and beautiful coastal heaths and woodlands were severely damaged.

Choosing suitable days for prescribed burning is difficult. Being too conservative may result in missed opportunities and the fuel reduction not being achieved. Attempting to burn on days that will not sustain a fire wastes resources. And prescribed burns that are too intense or escape carry significant consequences, not least because the public reaction to damage from a prescribed burn differs from that when the damage is due to a natural or accidental ignition.

Therefore we need to better understand occurrences of severe fire behaviour on days of apparently benign meteorology. Charney et al. (2003), Mills and Morgan (2006), Mills (2008a,b), Peace et al. (2012) and Peace and Mills (2012) have shown the importance of fine-scale meteorology to severe fire behaviour. This paper further contributes to this overall theme of the importance of fine-scale meteorology. However, while the theme is similar, the particular mesoscale atmospheric phenomenon is different - indeed, we present evidence that mountain waves and downslope flow, albeit on very modest topography, were crucial to the fire's escape.

\section{DESCRIPTION OF THE FIRE}

This summary of the fire draws heavily on Keelty (2012). Readers interested in greater detail are referred to that report.

The prescribed burn area was 722 ha in area and consisted of much of a rounded hill, $183 \mathrm{~m}$ high, which steeply slopes into the ocean to the west, and into Ellen Brook to the south. The southern boundary of this planned burn was Ellen Brook Road, a narrow bitumen road leading to a beachside car park. Heaths prevail close to the coast, grading to larger shrubs and small trees further inland. On the more sheltered eastern slopes are patches of tall karri forest, with a fairly dense understory. The age of the fuels varied, but was at least 30 years.

The procedure for prescribed burns is to first create a burnt edge to secure the burn, and then ignite the interior. This can be difficult in diverse fuels, since different vegetation types dry out and become ready to burn at different rates. "Edging" of the Ellenbrook burn was carried out on 6 September 2011, and resumed on November 10,11, 15, 16, 19 and 21, with attempts to ignite the interior on November 16, 19 and 21. It was difficult to achieve satisfactory ignition throughout this period, despite the use of progressively more aggressive methods from the ground and the air. By the end of the $21^{\text {st }}$, the edging remained incomplete in places, especially towards the southwest of the area, and there were large unburnt areas, of about 180 ha, in the centre and southwest of the area. The fire crews returned to the Ellenbrook burn on Tuesday 22 November, although without further attempts at ignition. By mid-afternoon, with the fires dying down, it was decided to not leave crews on duty overnight.

Witnesses reported smoke from the Ellenbrook fire from about 5 am WST (= UTC +8 hours) on Wednesday 23 November, and by 9.20 am the fire was in the car park at the western end of Ellen Brook Rd, outside of the prescribed area. At 10.20 am the Fire Operations Officer reported extreme fire behaviour with 20-m flames, and by noon the fire had crossed Ellen Brook Rd in numerous locations. The fire subsequently ran in a SSE direction across undulating terrain at an average speed of about $3 \mathrm{~km} / \mathrm{hr}$, crossing the Margaret River.

The fire diminished overnight, but resumed its southerly movement on the Thursday. It burnt out much of the remaining unburnt area north of the Margaret River and to the west of the previous day's scar, before again crossing the river, destroying the historic Wallcliffe House and burning through the Gnarabup community. It was eventually contained late that afternoon at Redgate Rd, south of Margaret River. 


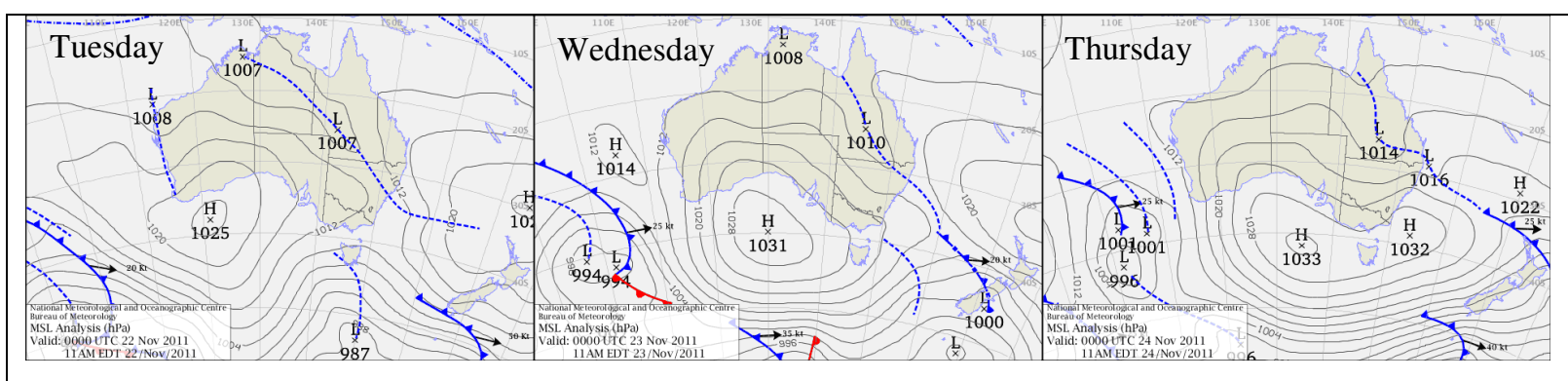

Fig. 1: Synoptic mean sea-level pressure charts valid at 8 am WST, for 24-hour intervals from Tuesday 22 November through to Thursday 24 November. From the Bureau of Meteorology.

\section{OVERVIEW OF SYNOPTIC-SCALE AND ANTECEDENT METEOROLOGY OF THE EVENT}

The antecedent conditions for this fire were that rainfall over the southwest of the state was about average for the spring of 2011, while both maximum and minimum temperatures were in the top decile for that period. Calculations of soil dryness indices (Keetch-Byram Drought Index and Soil Dryness Index) for a nearby automatic weather station (AWS) indicate that the dryness was close to the mean for the previous five years. These values, leading to a drought factor in the range of 7 to 8 , are consistent with those obtained from the Bureau of Meteorology's gridded analyses (Finkele et al. 2006) of these quantities.

Mean sea-level pressure (MSLP) analyses at 24-hour intervals of the fire period are shown in Fig. 1. On Tuesday $22^{\text {nd }}$, a high had moved into the Bight and was slowly strengthening. By the Wednesday morning, a slow-moving complex low pressure system had established to the southwest of the state, while the high pressure system continued to intensify. The resulting increase in the pressure gradient directed a markedly warmer and drier northerly airstream over the southwest of the state during the Wednesday and Thursday, eventually weakening and tending more north-westerly later on the Thursday as a cold front approached.

The behaviour of the fire during the day on the Wednesday and Thursday, once it had escaped, is broadly consistent with the shift to stronger, warmer and drier northerly winds. However, some unexplained features remain. The most important of these is the period from Tuesday afternoon through to Wednesday morning, during which the fire, which had been reluctant to burn and was dying down, reinvigorated and burnt into the hitherto unburnable fuels in the southwest of the prescribed area, before crossing Ellen Brook Road on Wednesday morning. The question of why the fire became active during this period will be the main focus of this article.

\section{THE MODEL}

The modelling strategy closely follows that successfully used by Engel et al. (2013) and Fawcett et al. (2013a) for their analyses of the Black Saturday fires in Victoria on 7 February, 2009, and by Fawcett et al. (2013b) for their investigation of the 1983 Melbourne duststorm. The atmospheric model is version 7.5 of the Unified Model developed at the UK MetOffice, as implemented by the Australian Bureau of Meteorology. The initial condition was the operational global analysis prepared by the Bureau valid at 11 am WST on Tuesday 22 November 2011. The model was run at successively finer nests, beginning with an approximately $40-\mathrm{km}$ mesh on a global domain, and proceeding through grid spacings of $11 \mathrm{~km}, 4 \mathrm{~km}, 1.2 \mathrm{~km}$ and $400 \mathrm{~m}$ on progressively smaller domains. There were 50 vertical levels, with the lowest few at approximately 20, 80, 180 and $320 \mathrm{~m}$ above the surface. Only results from the last domain, which was roughly square with sides of $300 \mathrm{~km}$, centred on the fire, are analysed here. The model results were compared against a range of surface and upper air observations, with generally satisfactory results, which will be reported in a longer paper currently in preparation.

\section{THE DETAILED METEOROLOGY}

\subsection{Overnight drying}

Beginning from about 2 am (1800 UTC) on the Wednesday, the air along the fire ground became noticeably drier. Initially this drier air was located along the northern part of the Leeuwin-Naturaliste block, but it migrated southwards through the early hours of the morning as the surface winds tended from easterly to north-easterly, until by 8 am it extended from the fire ground to south of Margaret River. The magnitude of the drying was that the surface dewpoint temperature over the fire ground fell to about $5^{\circ} \mathrm{C}$, and the relative humidity to about $30 \%$. 


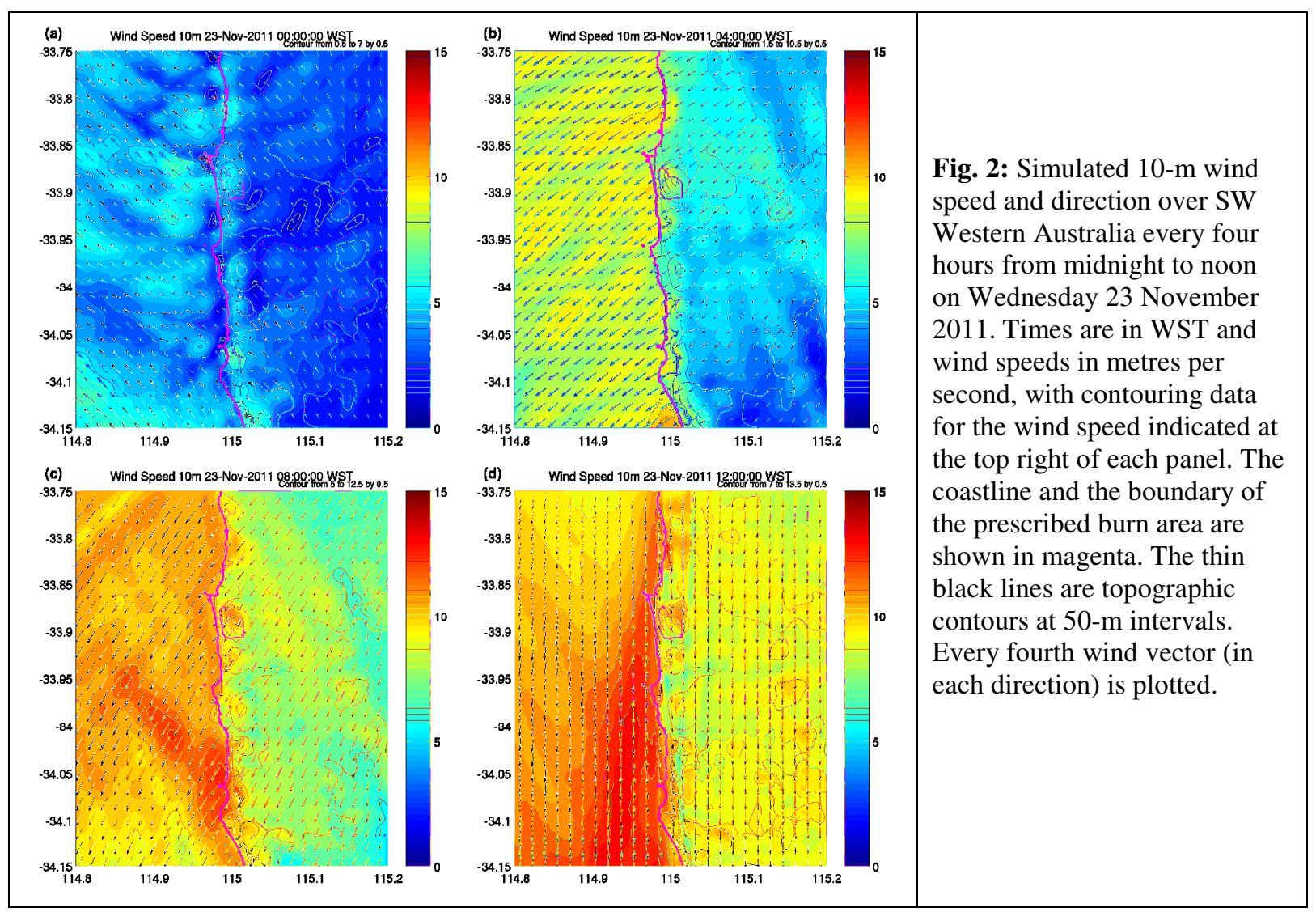

Fine fuels respond on time-scales of the order of an hour to changes in atmospheric moisture, and drying can significantly increase the flammability of fuel.

This drying is contrary to the normal diurnal cycle, in which the surface humidity is higher at night. The model simulation does not show evidence of significant subsidence, and nor would one normally expect such on the eastern flank of an approaching low pressure system. Rather, the simulation suggests that the dry air was advected in from inland areas to the northeast of the fire ground, where there was much drier air present, as the synoptic-scale flow strengthened and tended north-easterly. Examination of surface observations confirms both the drying, and its source. Reductions in surface humidity have also been studied by Mills (2008a,b) and related to severe bushfires, although the drying mechanism is different here.

\subsection{Surface winds}

By late Tuesday afternoon, the winds were a light southerly over the fire ground, which would have provided little support for the fire and is consistent with reports of low activity. However, the wind then strengthened and shifted south-easterly, consistent with the movement of the high pressure system. Figure 2a shows the simulated winds at midnight, in which it is apparent that the strongest surface winds over land occur in the immediate vicinity of the Leeuwin-Naturaliste coast, where they are about twice as strong as those inland. This pattern appeared from about $8 \mathrm{pm}$, and persisted through the night.

After midnight, the flow shifted to the east and then the northeast as the high moved further into the Bight. The winds strengthened inland, and also over the coastal fringe. From about 4 am on Wednesday the Ellenbrook fireground was in a local wind-speed maximum on the lee slopes of the hill (Fig. 2b). This local maximum strengthened over the next few hours, becoming especially marked from about $7 \mathrm{am}$. Figure $2 \mathrm{c}$ shows the surface winds at $8 \mathrm{am}$, and it is clear that the strongest winds over land during this period were collocated with the prescribed burn. The strong winds over the fire ground persisted through the morning. (The stronger winds over the ocean are consistent with the lower surface roughness there.) 


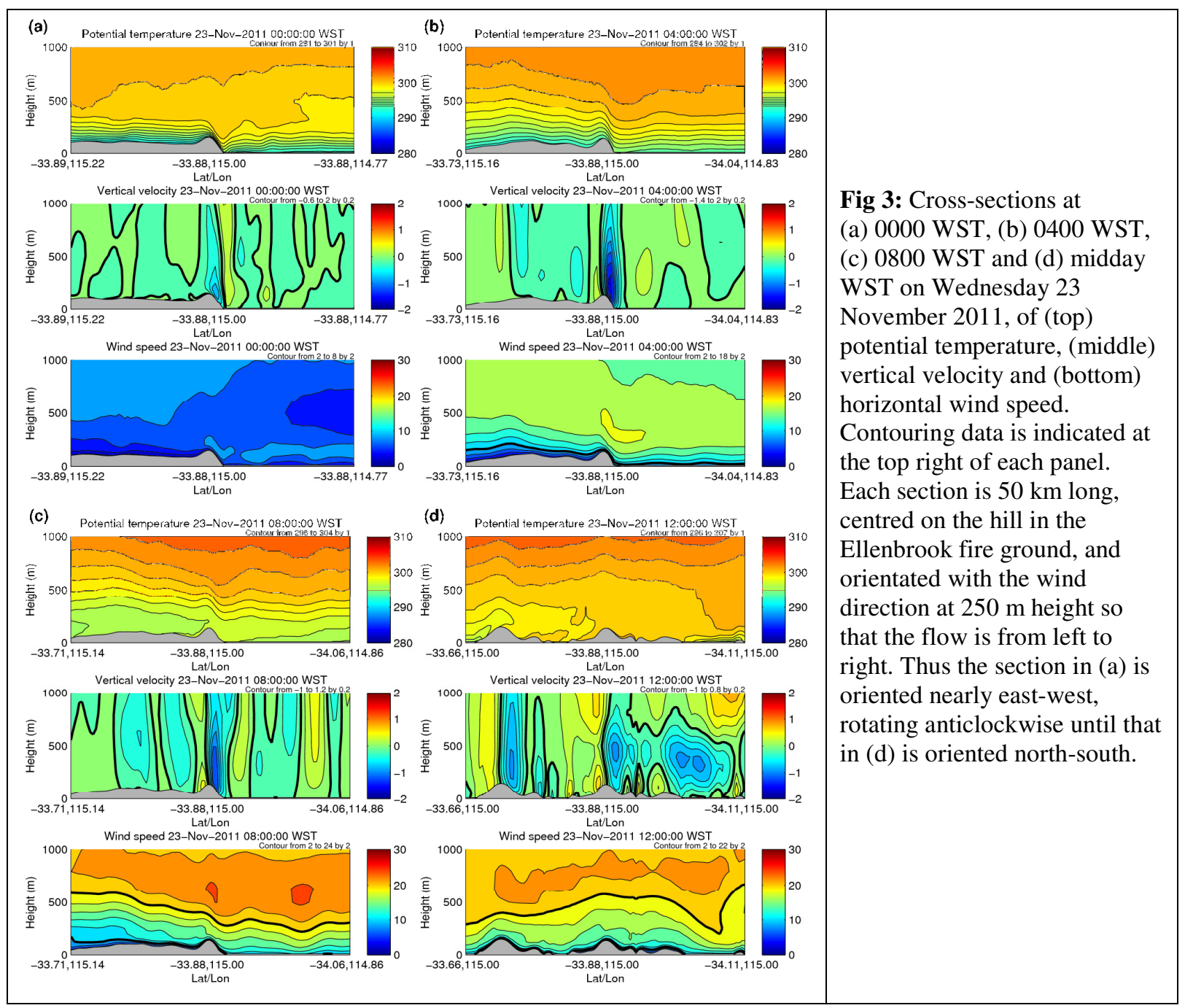

\subsection{Mountain waves}

Figure 3 shows cross-sections of potential temperature, vertical velocity and horizontal wind speed at 4-hour intervals from midnight Tuesday to midday Wednesday. The cross-sections are each $50 \mathrm{~km}$ long, centred on the hill, aligned along the wind direction at 250-m altitude (a little above the hill top), which rotates from approximately east-west at midnight, to north-south at midday.

At midnight on Tuesday, a marked nocturnal boundary layer exists over land, with a similar but moister and slightly weaker stable layer apparent over the sea. The isentropes dip steeply in the lee of the hill, being somewhat steeper than the topography. This dip reflects the marked descent on the lee slope, strongest near the surface, and an upwards rebound immediately offshore, giving the appearance of a hydraulic jump. The horizontal wind speed maximum similarly follows the slope downwards.

By 4 am on the Wednesday morning, the flow has tended more north-easterly and strengthened, and the surface stable layer is weaker. The downdraft on the hill's lee has strengthened substantially and extends much higher into the atmosphere, but the slope of the isentropes there has reduced. A marked low-level jet has developed, at about $600-700 \mathrm{~m}$ altitude upstream of the hill, reducing to $400 \mathrm{~m}$ downstream. The cause of this jet is still being investigated, but it does not appear to be a classical nocturnal jet.

By $8 \mathrm{am}$, when the downslope wind speeds were close to their peak, daytime heating over the land has eroded the nocturnal inversion and a layer 200 - $300 \mathrm{~m}$ deep of approximately constant potential temperature has developed, with a stable internal boundary layer apparent downwind of the hill over the sea. The low-level jet has strengthened to over $20 \mathrm{~m} / \mathrm{s}$, with less height difference between land and sea than earlier. The strong descent to the lee of the hill persists, with a second wave apparent some $15 \mathrm{~km}$ downwind. 
At midday, the mixed layer over the land has deepened and the stable layer aloft weakened. The stable layers over the sea are quite marked. The vertical motion associated with the hill has weakened somewhat, and there is no longer any indication of lee waves. The strongest winds have shifted towards the tops of the hills.

In summary, from Tuesday evening to Wednesday morning, there was marked mountain wave activity on the rather modestly sized hill that occupied much of the Ellenbrook prescribed burn area. This activity varied in detail - at times the simulation suggests a hydraulic jump, and at others weak lee waves. The effect was, however, a small area of strong north-easterly winds, directly over the unburnt fuels in the southwest of the burn area, which would have intensified the fire and pushed it directly towards the weak edge adjacent to the west end of Ellen Brook Rd. These winds persisted for a few hours after sunrise on Wednesday, and would have made it harder for the fire crews to contain the escape. Later in the day, with the loss of the near-surface stable layer, the mountain wave activity ceased and the wind maxima moved from the lee to the tops of the hills.

Given the fairly gentle slopes upstream, the contrasting steep slope downstream, and the importance of the nocturnal surface cooling, it appears that the downslope winds may be similar to the well-known Adelaide Gully Wind (Grace and Holton 1988) and the similar winds known to occur in the eastern suburbs of Perth on nights with strong easterlies.

\section{DISCUSSION}

The following meteorological factors likely contributed to the fire becoming active from the early morning of the 23rd onwards:

- Dry air over the fire ground from about $2 \mathrm{am}$.

- $\quad$ Strong downslope winds in the lee of the hills over the prescribed burn areas for much of Tuesday night and Wednesday morning.

- The development of a marked low level wind maximum during the night, which persisted through much of the day, together with transfer of the jet momentum down to the surface, initially by mountain wave activity and mechanical turbulence, and after sunrise by heating-induced turbulence also. A somewhat similar role for a low-level jet was suggested by Charney et al. (2003) to have contributed to the Mack Lake fire.

The simulations show that the Ellenbrook prescribed burn area may have been meteorologically the worst location along that coast to have a fire that morning, due to the combination of the overnight drying and the strong downslope winds. We propose that these factors were sufficient to transform the prescribed burn from being barely flammable one day, to uncontrollable the next.

This research suggests some limitations of current operational practice. The use of a daily maximum fire danger index will inevitably fail to detect those rare nights on which the fire danger drops only slightly, or not at all. The use of weather data representative of a large area will similarly obscure those small patches where the fire danger is much higher. Indeed, detecting such areas is also a challenge for forecasters: the strong downslope winds were not resolved in the coarser-resolution simulations, and much of the extant mountain-wave theory is for inviscid flow, a reasonable assumption for large mountains but not here, where the hill lay entirely within the boundary layer. Hence forecasting the occurrence of such winds at arbitrary locations will be a challenge. While our present knowledge is unfortunately insufficient to provide reliable forecasts of the occurrence and nonoccurrence of such winds, we do know that the basic ingredients of strong near-surface stability and reasonably strong gradient winds are not rare. We therefore recommend that meteorological and fire practitioners exercise a high degree of caution with fires on lee slopes, especially overnight.

\section{ACKNOWLEDGEMENTS}

This research was partly supported by the Bushfire Cooperative Research Centre. We acknowledge helpful discussions with Lachie McCaw and other DEC personnel, Ralph Smith, Mika Peace, Will Thurston and Kevin Tory.

\section{REFERENCES}

Charney, J.J., X. Bian, B.E. Potter and W.E. Heilman, 2003: Low-level jet impacts on fire evolution in the Mack Lake and other severe wildfires. In $2^{\text {nd }}$ International wildland fire ecology and fire management conference: $5^{\text {th }}$ symposium on fire and forest meteorology. Orlando, FL, U.S.A., November $16-20$.

Engel, C.B., T.P. Lane, M.J. Reeder and M. Rezny, 2013: The meteorology of Black Saturday. Quart. J. Roy. Meteorol. Soc., 139, 585 - 599. 
Fawcett R.J.B., Thurston W., Kepert J.D. and Tory K.J. 2013a. The meteorology of Black Saturday: a highresolution ACCESS modelling study. CAWCR Technical Report (in preparation).

Fawcett, R.J.B, A. Wain, W. Thurston, J.D. Kepert and K.J. Tory, 2013b: A comparison of the fire weather characteristics of the Melbourne dust storm (1983) and Black Saturday (2009): a high-resolution ACCESS case study. Papers presented at the $20^{\text {th }}$ ModSim Conference, Adelaide, Australia, December 1-6.

Finkele K, Mills G.A., Beard G. and Jones D.A. 2006. National daily gridded soil moisture deficit and drought factors for use in prediction of Forest Fire Danger Index in Australia. BMRC Research Report No. 119, Bureau of Meteorology, Australia.

Grace, W. and I. Holton, 1988: A mechanism for downslope winds with special reference to the Adelaide Gully Wind. Meteorological Note 179, Bureau of Meteorology, Australia.

Keelty M.J. 2012. Appreciating the Risk: Report of the Special Inquiry into the November 2011 Margaret River Bushfire. Government of Western Australia. 97pp.

Mills G.A. 2008a. Abrupt surface drying and fire weather Part 1: overview and case study of the South Australian fires of 11 January 2005. Australian Meteorological Magazine, 57, 299-309.

Mills G.A. 2008b. Abrupt surface drying and fire weather Part 2: a preliminary synoptic climatology in the forested areas of southern Australia. Australian Meteorological Magazine, 57, 311-328.

Mills G.A. and Morgan E 2006. The Winchelsea Convergence - using radar and mesoscale NWP to diagnose cool change structure. Australian Meteorological Magazine, 55, 47-58.

Peace M., McCaw L. and Mills G.A. 2012. Meteorological dynamics in a fire environment; a case study of the Layman prescribed burn in Western Australia. Australian Meteorological and Oceanographic Journal, 62, 127142.

Peace M. and Mills G.A. 2012. A case study of the 2007 Kangaroo Island bushfires. CAWCR Technical Report No. 53. Bureau of Meteorology, Australia.

Sharples J. 2009. An overview of mountain meteorological effects relevant to fire behaviour and bushfire risk. Int. J. Wildland Fire, 18, 737 - 754. 